\title{
HERMITIAN FORMS. I $\left({ }^{1}\right)$
}

\author{
BY \\ H. P. $\operatorname{ALLEN}\left({ }^{2}\right)$
}

The present paper studies the classification problem for hermitian forms. We follow the approach used by Springer in [3] in discussing the quadratic form case. Our methods indicate new avenues of study for unitary groups of type $\mathrm{D}$. The first parts are devoted to developing relationships between sesquilinear forms, involutions, and quadratic forms (over suitable extensions). This is applied to the last part where we pursue the equivalence question in terms of cohomological invariants. In what follows, all fields will have characteristic unequal to 2 .

1.1. Let $\Delta$ be an associative division algebra, finite dimensional over its center $k$, - an involution in $\Delta / k, E$ an $m$-dimensional right vector space over $\Delta$, and $f$ a nondegenerate ${ }^{-}$sesquilinear form on $E$. Thus $f$ is a biadditive mapping from $E \times E$ to $\Delta$ which is linear in the second variable and -linear in the first $\left({ }^{3}\right) . f$ is called hermitian or skew-hermitian (- being understood) as

$$
\overline{f(y, x)}=f(x, y) \quad \text { or } \quad-f(x, y) \text { for all } x, y \in E .
$$

For this section, we will use the word form to represent either a-hermitian or - skew-hermitian form (always nondegenerate).

It is well known that a form $f$ defines an involution $J$ in $\mathfrak{A}=\operatorname{Hom}_{\Delta}(E, E)$ which is characterized by the relation

$$
f(x T, y)=f\left(x, y T^{J}\right), \quad x, y \in E, T \in \mathfrak{A} .
$$

The converse is also true, for we have

Proposition 1. Let $\mathfrak{A}=\operatorname{Hom}_{\Delta}(E, E)$ where $\Delta$ is a central division algebra over $k$, and let - be any involution in $\Delta / k$. If $J$ is any involution in $\mathfrak{X} / k$ then there is a form on $E$ relative to which $J$ is the adjoint. Moreover if $f$ and $g$ are forms on $E$ (-bilinear) which have the same adjoint, then $f=\lambda g$ for some $\lambda \in k^{*}$.

Proof. Let $\left(e_{1}, \ldots, e_{m}\right)$ be a basis for $E$ and let $g$ be the -hermitian form $g(x, y)=\sum \bar{a}_{i} \beta_{i}, x=\sum e_{i} \alpha_{i}, y=\sum e_{i} \beta_{i}$. If $K$ is the adjoint mapping determined by $g$, then it is easy to see that there is a unit $A \in \mathfrak{A}$ where $A^{K}=\varepsilon A, \varepsilon=1$, or -1 so that

Received by the editors December 12, 1966 and, in revised form, April 22, 1968

( ${ }^{1}$ This research was supported in part by National Science Foundation Grant GP 4361.

(2) Part of this research was conducted while the author was a NATO postdoctoral research fellow.

$\left(^{3}\right)$ Usually one requires that $f$ be linear in the first variable and - linear in the second. This is only a notational difference since we are emphasizing the "right" vector space structure. 
$T^{J}=A^{-1} T^{K} A$ for all $T \in \mathfrak{A}$. It is immediate that $J$ is then the adjoint mapping with respect to the $\varepsilon$-hermitian form $f(x, y)=g\left(x, y A^{-1}\right)$.

Now suppose that $f$ and $g$ are forms on $E$ which have the same adjoint, say $J$. By nondegeneracy, there is an $A \in \mathfrak{U}$ with $f(x, y)=g(x, y A)$. Using the fact that $J$ is the adjoint with respect to both forms we conclude that $A$ is in the center of $\mathfrak{A}$ viz., $k$. Since $A$ is clearly invertible, we obtain the stated conclusion. Q.E.D.

An involution $J$ in $\mathfrak{A} / k$ is said to be of Type $\mathrm{C}$ or $\mathrm{D}$ depending on whether $[\mathfrak{s}(\mathfrak{A}, J): k]=\frac{1}{2} n(n+1)$ or $\frac{1}{2} n(n-1)$ where $\mathfrak{s}(\mathfrak{A}, J)$ is the Lie algebra of all $J$-skew elements of $\mathfrak{A}$ and $[\mathfrak{A}: k]=n^{2}$. Equivalently, if $\bar{k}$ is an algebraic closure of $k$, then the linear extension of $J$ to $\mathfrak{A} \otimes_{k} \bar{k}$ is cogredient to either the adjoint mapping of a nondegenerate alternate form or a nondegenerate quadratic form depending on whether the type is $\mathrm{C}$ or $\mathrm{D}$. A more explicit criterion in terms of $f$ is given in

THEOREM 1. Let $f$ be a form on $E / \Delta, \mathfrak{A}=\operatorname{Hom}_{\Delta}(E, E)$ and let $J$ be the adjoint in $\mathfrak{A}$ determined by $f$. Then $J$ is of type $\mathrm{D}$ if $f$ is hermitian and ${ }^{-}$is of type $\mathrm{D}$ or if $f$ is skew-hermitian and - is of type $\mathrm{C}$. In all other cases the type is $\mathrm{C}$.

Proof. Choose a basis $\left(e_{1}, \ldots, e_{m}\right)$ for $E$ and let $g$ be the form $g(x, y)=\sum \bar{a}_{i} \beta_{i}$. By Proposition 1, there is an invertible element $A \in \mathfrak{A}$ where $A^{K}=\varepsilon A, \varepsilon=1$ or -1 with $f(x, y)=g\left(x, y A^{-1}\right)$. We let $K$ be the adjoint corresponding to $g$ and observe that $K$ is of type $\mathrm{C}$ if and only if - is of type $\mathrm{C}$, for relative to the above basis, $K$ is -transpose in $\Delta_{m}$.

Left multiplication by $A$ induces a linear isomorphism between $\mathfrak{s}(\mathfrak{A}, J)$ and $\mathfrak{s}(\mathfrak{A}, K)$ if $A$ is $K$-symmetric whereas if $A$ is $K$-skew, we obtain a linear isomorphism between $\mathfrak{s}(\mathfrak{A}, J)$ and $\mathfrak{h}(\mathfrak{A}, K)$, the subspace of all $K$-symmetric elements of $A$. Since $f$ is hermitian if and only if $\varepsilon=1$, we can read off the above assertions directly. Q.E.D.

Now let - and ${ }^{\wedge}$ be two involutions in $\Delta / k$. By the above results, there is an $a \in \Delta^{*}$ with $\bar{a}= \pm a$ and $\hat{b}=a^{-1} \bar{b} a$ for all $b \in \Delta$. If $f$ is either a hermitian or skew-hermitian form, then $g=a f$ is a -hermitian or -skew-hermitian form. It is clear that both $f$ and $g$ determine the same involution in $\mathfrak{A}$. Thus we may fix a single involution, say - , in $\Delta / k$ and develop invariants for -forms. This will determine arbitrary forms, involutions in $\Delta / k$ varying, to within multiples, and also the associated involutions to within equivalence.

1.2. By way of illustrating some of the results which we will obtain, we will need some technical information about crossed product division algebras. Any unquoted result may be found in Albert [1].

Let $\Delta$ be a central division algebra over $k$ of dimension $r^{2}$. The integer $r$ is called the degree of $\Delta$. A subfield $P / k$ is called maximal if $[P: k]=r$. It is known that $\Delta$ always has a maximal (separable) subfield. If $P / k$ is any maximal subfield then $\Delta_{P}=\Delta \otimes_{k} P$ is split. Indeed, if we consider $\Delta$ as a left vector space over $P$ and equip $\Delta / P$ with a $\Delta_{P}$-module structure by defining

$$
d^{\prime} \cdot d \otimes \pi=\pi d^{\prime} d
$$


then we see that the associated representation $\Delta_{P} \rightarrow \operatorname{End}_{P} \Delta$ is an isomorphism.

$\Delta$ is called a crossed product algebra if it has a maximal subfield which is Galois over $k\left({ }^{4}\right)$. We briefly recall the structure of such algebras: if $P / k$ is maximal and Galois and $G=\operatorname{gal}(P / k)$, then there is a subset $\left\{T_{s} \mid s \in G\right\}$ of $\Delta$ such that $\Delta=$ $\sum \oplus T_{s} P$ (as a linear space) and multiplication is given by

$$
\begin{aligned}
\pi T_{s} & =T_{s} \pi^{s}, & & \pi \in P, s \in G, \\
T_{s} T_{h} & =T_{s h} \delta(s, h), & & s, h \in G, \delta(s, h) \in P^{*} .
\end{aligned}
$$

$(s, h) \rightarrow \delta(s, h)$ is a 2-cocyle of $G$ in $P^{*}$ whose cohomology class in $\mathscr{H}^{2}\left(G, P^{*}\right)$ is the image of the Brauer class of $\Delta^{-1}$ under the canonical isomorphism $\mathscr{B}(P / k) \rightarrow$ $\mathscr{H}^{2}\left(G, P^{*}\right)(\mathscr{B}(P / k)$ denoting the subgroup of the Brauer group over $k$ split by $P)$. One usually writes $\Delta=(P, G, \delta)$.

Without loss of generality we may assume that $\delta$ is normalized so that $T_{e}$ is the identity of $\Delta$ ( $e$ the identity of $G$ ). Assume now that $\Delta$ is involutorial. This is equivalent to saying that $\delta^{2}$ is a coboundary. Thus we have a 1-cocycle $\gamma$ with

$$
\delta^{2}(s, h)=\gamma(s h)^{-1} \gamma(s)^{h} \gamma(h) \quad(\text { note } \gamma(e)=1) .
$$

We use the above splitting to define a map ${ }^{-}$in $\Delta / k$ by the rule

$$
\sum T_{s} \pi_{s} \rightarrow \overline{\sum T_{s} \pi_{s}}=\sum \pi_{s} \gamma(s) T_{s}^{-1} .
$$

It is easy to verify that - is an involution, in fact, it is an involution of type D. To see the latter write $G$ as the disjoint union $G=\{e\} \cup I \cup W \cup W^{-1}$ where $I$ is the set of all elements of order 2 different from $e$. For $s \in I, T_{s} P$ is - stable and for $s \in W, T_{s} P+T_{s^{-1}} P$ is - stable, and we let $I_{s}$ and $W_{s}$ be the subspaces of the above of all ${ }^{-}$symmetric elements. Then $\mathfrak{h}(\Delta,-)$, the subspace of all - symmetric elements of $\Delta$, is the direct sum

$$
\mathfrak{h}(\Delta,-)=T_{e} P \oplus \sum I_{s} \oplus \sum W_{s} .
$$

It is clear that $\left[W_{s}: k\right]=[P: k]=r$. If $s \in I$ and $P_{s}$ is the fixed field of $s$, then a simple calculation shows that $\left[I_{s}: k\right]=\left[P_{s}: k\right]=\frac{1}{2} r$. Thus if $|I|=\xi$, and $|W|=\eta$, then $r-1=\xi+2 \eta$, and $[\mathfrak{h}(\Delta,-): k]=r+\xi \frac{1}{2} r+\eta r=\frac{1}{2} r(r+1)$, as claimed. An involution in $\Delta / k$ of the form given by (4) will be called standard.

Observe that if $\Delta$ is a quaternion division algebra, that the involutions which arise from the composition algebra structure, and which are referred to in the literature as "standard", are not standard in the above sense. Indeed, a "standard" involution is clearly of type C.

Any division algebra of degree 2 is cyclic, so if $\Delta$ is a quaternionic division algebra, $P$ a maximal subfield (necessarily Galois), $\{e, t\}=\operatorname{gal}(P / k)$, then there is a $T \in \Delta$ where $T^{2}=\delta \in k^{*}, \Delta=P \oplus T P$ and $\pi T=T \pi^{t}$ for all $\pi \in P$. The factor set is

$\left.{ }^{4}\right)$ It is not known whether or not every finite dimensional central simple associative division algebra is a crossed product. 
$\delta(e, e)=\delta(e, t)=\delta(t, e)=1, \delta(t, t)=\delta$. The 1-cocycle $e \rightarrow 1, t \rightarrow \delta$ has coboundary $\delta^{2}$, so the standard involution determined from these ingredients is $\alpha+T \beta \rightarrow \alpha+T \beta^{t}$.

2.1. We restrict ourselves now to involutions of type D. Since every involutorial division algebra always has an involution of type $\mathrm{D}$, the discussion in $\$ 1.1$ shows that we may realize an involution of type $D$ as the adjoint mapping relative to some -hermitian form, - a fixed involution of type $\mathrm{D}$ in $\Delta$. If $\Delta$ is a crossed product algebra, then - will always represent a standard involution.

Let $f$ be a hermitian form on $E, J$ the corresponding adjoint mapping and let $P / k$ be a maximal subfield of $\Delta$. We consider $E$ as a vector space over $P \subset \Delta$ by restriction of scalars and by an argument analogous with that given in $\S 1.2$, we obtain a natural identification between $\mathfrak{A}_{P}$ and $\operatorname{End}_{P} E / P\left(\mathfrak{A}=\operatorname{Hom}_{\Delta}(E, E)\right)$. If we let $J$ also denote the involution $J \otimes 1$ in $\operatorname{End}_{P} E / P$, then Proposition 1 shows that there is a quadratic form $q$ on $E / P$ having $J$ as its adjoint.

We shall adopt without further mention the following convention: let $g$ be a fixed -hermitian form on $E$ and choose a nondegenerate symmetric bilinear form $g_{P}$ on $E / P$ which has $K \otimes 1 \equiv K(K$ the adjoint associated to $g$ ) as its adjoint as indicated above. Let $f$ be an arbitrary hermitian form on $E$. As in the proof of Theorem 1, there is an invertible $K$-symmetric element $A \in \mathfrak{A}$ with $f(x, y)=$ $g\left(x, y A^{-1}\right)$ for all $x, y \in E$. Let $f_{P}(x, y)=g_{P}\left(x, y A^{-1}\right)$ (we are identifying $\mathfrak{A}$ with $\mathfrak{A} \otimes 1 \subseteq \operatorname{End}_{P} E / P$ since this is consistent with their actions on $E / \Delta$ and $E / P$ respectively). If $J$ is the adjoint with respect to $f$, then we have $T^{J}=A^{-1} T^{K} A$ for all $T \in \mathfrak{A}$. This shows that $J(\equiv J \otimes 1)$ is the adjoint with respect to $f_{P}$. The reason for this normalization is that we want the mapping $f \rightarrow f_{P}$ to preserve equivalence. Indeed if $f_{1}$ is a -hermitian form on $E$ and $T \in \mathfrak{A}$ an equivalence between $f$ and $f_{1}$ $\left(f(x T, y T)=f_{1}(x, y)\right)$, then $f_{1}(x, y)=g\left(x, y A_{1}^{-1}\right)$ where $A_{1}^{-1}=T A^{-1} T^{K}$. Then $f_{1 P}(x, y)=g_{P}\left(x, y A_{1}^{-1}\right)=f_{P}(x T, y T)$.

If $\Delta$ is the crossed product algebra $(P, G, \delta)$, then we can construct " $f_{P}$ " as follows: here ${ }^{-}$is the standard involution defined by (4). For $x, y \in E$ write

$$
f(x, y)=\sum T_{s}(x, y)_{s} \text {. }
$$

Each $(,)_{s}$ is a nondegenerate $s$-sesquilinear form on $E / P$. The conditions that $f$ is - hermitian, linear in the second variable, and -linear in the first are equivalent to

$$
\begin{aligned}
& (x, y)_{s}=\delta\left(s^{-1}, s\right)^{-1} \gamma\left(s^{-1}\right)^{s}(y, s)_{s}^{s}-1 \quad \text { for } s \in G, \\
& \left(x, y T_{h}\right)_{s}=\delta\left(s h^{-1}, h\right)(x, y)_{s h}^{h}-1 \quad \text { for } s, h \in G, \\
& \left(x T_{h}, y\right)_{s}=\delta(h, s)^{-1} \gamma(h)^{s}(x, y)_{h s} \quad \text { for } h, s \in G,
\end{aligned}
$$

respectively.

(5) shows that $(,)_{e}$ is a symmetric nondegenerate bilinear form on $E / P$, and $f(x T, y)=f\left(x, y T^{J}\right)$ shows that (the linear extension of ) $J$ is the adjoint with respect to $(,)_{e}$. To see that this is consistent with our convention, observe that if we write $g(x, y)=\sum T_{s}(x, y)_{s}^{\prime}$, and choose $g_{P}$ as $(,)_{e}^{\prime}$, then $f(x, y)=g\left(x, y A^{-1}\right)$ implies that 
$(x, y)_{e}=\left(x, y A^{-1}\right)_{e}^{\prime}=f_{P}(x, y)$. When dealing with crossed product division algebras we will always make the above choice of $f_{P}$.

REMARK. We have shown elsewhere (Hermitian forms. II, forthcoming) that $f(x, y)=0 \Rightarrow f_{P}(x, y)=0$ (no assumption on $P$ ), although the converse is not true. We call a form $f P$-rigid if there is an "extension" $f_{P}$ which satisfies the condition, $f(x, y)=1 \Rightarrow f_{P}(x, y)=1$. It then follows that $f(x, y)=f_{P}(x, y)$ whenever it makes sense. It is easy to see that if there is a $P$-rigid form, then every form is $P$-rigid. Seip-Hornix has recently shown that all forms are $P$-rigid (no restriction on $P$ ). This is obvious when $P$ is a Galois extension (cf. above formula) and for suitable $P$, one can show that all forms are $P$-rigid by explicitly constructing $f_{P}$ from $f\left({ }^{5}\right)$. Using the fact that the Jordan algebra $\mathfrak{h}\left(\Delta,{ }^{-}\right)$is unramified, we obtain the existence of a maximal separable subfield $P / k$ which is - symmetric. By a simple argument using Galois descent, one easily sees that the generic trace $(\operatorname{tr}(\cdot))$ on $\Delta$, when restricted to the subspace $P \subseteq \Delta$, reduces to the usual field-theoretic trace $(t(\cdot))$. For $x, y \in E, \pi \in P, \pi \rightarrow \operatorname{tr}(f(x, y) \pi)$ is a linear functional on $P / k$ and hence there exists a unique element in $P$, which we denote by $f_{P}(x, y)$, such that

$$
\operatorname{tr}(f(x, y) \pi)=t\left(f_{P}(x, y), \pi\right)
$$

for all $\pi \in P$. Using the fact that $P$ is ${ }^{-}$symmetric one sees that $f_{P}$ is a nondegenerate bilinear form on $E / P$ whose associated adjoint is (the linear extension of) the adjoint corresponding to $f$ and that $f_{P}$ satisfies the requirement of rigidity. It is also clear that this choice of $f_{P}$ is consistent with our convention.

2.2. Assume now that $P / k$ is a maximal separable subfield and let $L / k$ be a finite dimensional Galois extension containing $P$. Let $f$ be a -hermitian form on $E$ and let $f_{L}$ be the linear lift of $f_{P}$ to a nondegenerate form on $V=(E / P)_{L} \cdot \mathfrak{A}$ is a $k$-algebra form of $\operatorname{End}_{L} V$ and it is clear that the linear lift of $J$, the adjoint with respect to $f$, is the adjoint with respect to $f_{L}$ (we continue to denote this involution as $J)$. For $s \in G=\operatorname{gal}(L / k)$, the endomorphism $\eta(s)=1 \otimes s$ in $\operatorname{End}_{L} V=\mathfrak{A} \otimes_{k} L$ is an $s$-linear automorphism which commutes with $J$. It is easy to see that $\eta(s)$ has the form $T \rightarrow t(s)^{-1} T t(s)$ where $t(s)$ is an $s$-linear transformation on $V$ satisfying $t(s) t(h)=t(s h) \delta(s, h), \delta(s, h) \in L^{*} .(s, h) \rightarrow \delta(s, h)$ is a 2-cocyle whose cohomology class is the class corresponding to $\Delta$ ( $\Delta$ is split by $L$ ). Since $\eta(s)$ commutes with $J$, $t(s)$ is an $s$-linear similitude of $f_{L}$, of ratio say $\gamma(s)\left(f_{L}(x t(s), y t(s))=\gamma(s) f_{L}(x, y)^{s}\right)$. By our choice of $f_{P}$, hence also $f_{L}$, the ratio $\gamma(s)$ is the same for all -hermitian forms on E.

Let $\mathbb{Q}$ be the set of all nondegenerate symmetric bilinear forms on $V$ and define an action of $G$ on $\mathfrak{Q}$ by setting

$$
b^{s}(x, y)=\gamma(s) b\left(x t(s)^{-1}, y t(s)^{-1}\right)^{s}
$$

${ }^{(5)}$ I am indebted to T. A. Springer for suggesting this choice of $f_{P}$. 
It is easily verified that this action equips $\mathscr{D}$ with the structure of a $G$-set (observe that $\left.\delta(s, h)^{2}=\gamma(s h)^{-1} \gamma(s)^{h} \gamma(h)\right)$. Since each $t(s)$ is an $s$-linear similitude of ratio $\gamma(s)$ of every $f_{L}$ we have half of

THEOREM 2. The correspondence $f \rightarrow f_{L}$ is a bijection between the set of all

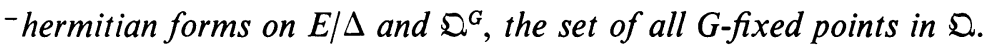

Proof. The correspondence is clearly injective since $f \rightarrow f_{P}$ is by definition injective. To see that it is surjective, let $b \in \mathfrak{Q}^{G}$ and let $g$ be the ${ }^{-}$hermitian form on $E$ which was used to formulate our convention at the beginning of $\$ 2.1$. We may write $b(x, y)=g_{L}\left(x, y A^{-1}\right)$ where $A$ is a $g_{L}$-symmetric invertible element of $\operatorname{End}_{L} V$. Since $b$ is fixed under $G$, we see that $A$ commutes with each $t(s)$ and this implies that $A \in \mathfrak{A}$. If we define a ${ }^{-}$hermitian form on $E$ by $f(x, y)=g\left(x, y A^{-1}\right)(A$ is now necessarily $g$-symmetric), then it is clear from the definitions involved that $b=f_{L}$. Q.E.D.

REMARK. If $L=P$, so that $\Delta$ is the crossed product algebra $(P, G, \delta)$, then we can obtain the last part of the above proof by explicitly constructing $f$ from $b$ (previous notations in effect). Indeed, if we set $(x, y)_{s}=b\left(x, y T_{s}^{-1}\right)^{s}$ (note that $T_{s}=t(s)$, and that the $\gamma(s)$ are the same as in (4)), then a straightforward computation shows that

$$
f(x, y)=\sum T_{s}(x, y)_{s}
$$

satisfies equations (5), (6), and (7) and thus is a nondegenerate -hermitian form on $E$. Clearly $b=(,)_{e}$.

2.3. It is clear that if $f_{1}$ and $f_{2}$ are two -hermitian forms on $E / \Delta$, then there is a finite dimensional Galois extension $L / k \supseteq P / k(P / k$ as in the last section) such that $f_{1 L}$ and $f_{2 L}$ are equivalent. Thus we fix a single -hermitian form $f$ and for each finite dimensional Galois extension $L / k \supset P / k$ we let $(L, f)$ be the set of all -hermitian forms $f_{1}$ on $E$ with $f_{1 L}$ equivalent to $f_{L}$. By our convention concerning the choice of the $f_{1 P}$ 's, it follows that $(L, f)$ is a union of equivalence classes of forms.

Let $G=\operatorname{gal}(L / k)$ and let $t(s), \gamma(s)$ and $\delta(s, h)$ be as described in $\S 2.2$. If $f_{1} \in(L, f)$ and $T \in \operatorname{End}_{L} V$ is an equivalence between them $\left(V=(E / P)_{L}\right)$, then for $s \in G$

$$
\begin{aligned}
f_{1 L}(x, y) & =f_{1 L}^{s}(x, y) \\
& =\gamma(s) f_{L}\left(x t(s)^{-1} T, y t(s)^{-1} T\right)^{s} \\
& =f_{L}^{s}\left(x T^{s}, y T^{s}\right) \\
& =f_{L}\left(x T^{s}, y T^{s}\right) .
\end{aligned}
$$

Thus $T$ and $T^{s}$ are both equivalences between $f_{L}$ and $f_{1 L}$ and this implies that $T^{s}=T U_{s}$ where $U_{s} \in O\left(f_{L}\right) . T^{s h}=\left(T^{s}\right)^{h}$ implies that $U_{s h}=U_{h} U_{s}^{h}$ so $s \rightarrow U_{s}$ is a 1-cocycle of $G$ in $O\left(f_{L}\right)$. Its 1-cohomology class in $\mathscr{H}^{1}\left(G, O\left(f_{L}\right)\right) \equiv \mathscr{H}^{1}(L, f)$, will be denoted by $c_{L}\left(f, f_{1}\right)$. We now have

THEOREM 3. The correspondence $f_{1} \rightarrow c_{L}\left(f, f_{1}\right)$ is well defined and induces a bijection between the set of equivalence classes in $(L, f)$ and $\mathscr{H}^{1}(L, f)$. 
Proof. It is easy to see that if we choose different equivalences between $f_{L}$ and $f_{1 L}$, then the 1-cocycles which arise differ by a coboundary and the map is thus well defined.

Let $f_{i} \in(L, f), i=1,2$, and let $T \in \mathfrak{A}$ be an equivalence between them. If $T_{1} \in$ End $_{L} V$ is an equivalence between $f_{L}$ and $f_{1 L}$ with $T_{1}^{s}=T_{1} U_{s}$, then $f_{L}\left(x T T_{1}, y T T_{1}\right)=$ $f_{1 L}(x T, y T)=f_{2 L}(x, y)$. Thus we can compute $c_{L}\left(f, f_{2}\right)$ from $T T_{1}$. Since $T \in \mathfrak{A}$ we have $\left(T T_{1}\right)^{s}=T T_{1} U_{s}$, and this shows that $c_{L}\left(f, f_{1}\right)=c_{L}\left(f, f_{2}\right)$. Thus $c_{L}\left(f, f_{1}\right)$ depends only on the equivalence class represented by $f_{1}$. It remains to show that the correspondence is bijective.

Injective. Suppose that $c_{L}\left(f, f_{1}\right)=c_{L}\left(f, f_{2}\right)$. Then we can find equivalences $T_{i}$ between $f_{L}$ and $f_{i L}, i=1,2$, where $T_{i}^{s}=T_{i} U_{s}, i=1,2$. If we set $T=T_{1} T_{2}^{-1}$, then $T^{s}=T$ for all $s \in G$ and thus $T \in \mathfrak{A}$. $T$ is an equivalence between $f_{2 L}$ and $f_{1 L}$ and it follows (from our convention) that $T$ is also an equivalence between $f_{2}$ and $f_{1}$.

Surjective. Let $s \rightarrow U_{s}$ be a 1-cocycle of $G$ in $O\left(f_{L}\right)$. By a theorem of Springer [3, appendix], there is a $T \in \mathrm{GL}(V)$ with $U_{s}=T^{-1} T^{s}$. Define a nondegenerate symmetric bilinear form $b$ on $V$ by $b(x, y)=f_{L}(x T, y T)=f_{L}\left(x, y T T^{J}\right), J$ the adjoint with respect to $f_{L}$. A simple calculation shows that $b^{s}=b$ for all $s \in G$ and this implies that $T T^{J} \in \mathfrak{A}$ (and it is clearly $f$-symmetric). Let $f_{1}$ be the -hermitian form on $E$ defined by $f_{1}(x, y)=f\left(x, y T T^{J}\right)$. It is immediate that $b=f_{1 L}$ so $f_{1} \in(L, f)$. Since $T$ is an equivalence between $f_{L}$ and $f_{1 L}$, and by definition $T^{s}=T U_{s}$, we see that $c_{L}\left(f, f_{1}\right)$ is the cohomology class of $s \rightarrow U_{s}$. Q.E.D.

2.4. Let $N$ be the generic norm on $\mathfrak{A}$. Recall that $N$ is a multiplicative homogeneous mapping of degree $n=m r$ and is invariant under automorphisms and antiautomorphisms. If $\bar{k}$ is an algebraic closure of $k$, then for $a \in \mathfrak{A}, N(a)=$ $\operatorname{det}(a \otimes 1)$.

If $f$ is any hermitian form on $E$ and $\left(e_{1}, \ldots, e_{m}\right)$ is a right $\Delta$ basis for $E$, then the coset $N\left(f\left(e_{i}, e_{j}\right)\right) k^{* 2}$ is independent of the choice of basis. It follows from this that $f$ determines a unique element of $k^{*} / k^{* 2}$ which we denote by $d(f)$ and which we call the discriminant of $f$. In Hermitian forms. II, we show that if $m$ is even, then $\boldsymbol{d}(f)_{P}=\boldsymbol{d}\left(f_{P}\right)$, where $\boldsymbol{d}\left(f_{P}\right)$ is the usual discriminant for quadratic forms and $x \rightarrow x_{P}$ is the obvious mapping from $k^{*} / k^{* 2}$ to $P^{*} / P^{* 2}$.

LEMMA 1. The following are equivalent, $f_{1} \in(L, f)$ and

(a) there is an equivalence $T$ between $f_{L}$ and $f_{1 L}$, where $\operatorname{det} T \in k$;

(b) there is an equivalence $T$ between $f_{L}$ and $f_{1 L}$ where $T^{s}=T U_{s}, U_{s} \in O^{+}\left(f_{L}\right)$, for all $s \in G$;

(c) $d(f)=d\left(f_{1}\right)$.

Proof. It is clear that (a) and (b) are equivalent, since det is a $G$-map. Write $f(x, y)=g\left(x, y A^{-1}\right), f_{1}(x, y)=g\left(x, y A_{1}^{-1}\right)$ and let $T \in \operatorname{End}_{L} V$ be an equivalence between $f_{L}$ and $f_{1 L}$. We then obtain $A_{1}^{-1}=T A^{-1} T^{K}$ where $K$ is the adjoint with respect to $g_{L}$. Hence $(\operatorname{det} T)^{2}=\operatorname{det}\left(A A_{1}^{-1}\right)$. Since $f\left(x, y A A_{1}^{-1}\right)=f_{1}(x, y)$,

$$
d(f) N\left(A A_{1}^{-1}\right) k^{* 2}=d\left(f_{1}\right) .
$$


Since $N\left(A A_{1}^{-1}\right)=\operatorname{det}\left(A A_{1}^{-1}\right)$, we see that det $T \in k$ if and only if $d(f)=d\left(f_{1}\right)$. Thus (c) is equivalent to (a). Q.E.D.

We let $(L, f)^{+}$denote the subset of $(L, f)$ consisting of all forms having the same discriminant as $f$. The above lemma shows that the correspondence described in $\S 2.3$ induces a bijection between $(L, f)^{+}$and $\mathscr{H}_{+}^{1}(L, f) \equiv \mathscr{H}^{1}\left(G, O^{+}\left(f_{L}\right)\right)$.

In order to discuss the 2-cohomological invariants which we shall assign to hermitian forms we need the notion of the Jacobson even Clifford algebra of the form (Jacobson [2]). The details follow.

3.1. Let $\mathfrak{E}\left(f_{L}\right)$ be the Clifford algebra of $f_{L}$. Recall that $\mathfrak{S}\left(f_{L}\right)$ is the quotient algebra $\mathfrak{T}(V) / \mathfrak{\Im}$, where $\mathfrak{T}(V)$ is the tensor algebra on $V$ and $\mathfrak{\Im}$ is the ideal generated by $\left\{x \otimes x-f_{L}(x, x) \mid x \in V\right\}$. $\Im$ is generated by even elements so $\mathfrak{S}\left(f_{L}\right)$ has an evenodd gradation and we let $\mathfrak{C}^{+}\left(f_{L}\right)$ be the subalgebra of all even elements. By extending the base field, we see that $\mathbb{C}^{+}\left(f_{L}\right)$ is either the ideal direct sum of two central simple algebras or is simple with center a quadratic extension of $L$. The first case corresponds to $d\left(f_{L}\right)=1$ and in the second case, the center is obtained by adjoining a square root of $d\left(f_{L}\right)$ (note that $[V: L]$ is even). In terms of Galois descent, this is the distinction between type $\mathrm{D}_{\mathrm{I}}$ and type $\mathrm{D}_{\mathrm{II}}$ in Jacobson [2]. Finally, the exchange involution in $\mathfrak{T}(V) x \otimes y \otimes \cdots \otimes z \rightarrow z \otimes \cdots \otimes y \otimes x$ leaves $\Im$ stable and induces an involution in $\mathfrak{S}\left(f_{L}\right)$ which we denote by $\iota$. $\iota$ leaves $\mathfrak{C}^{+}\left(f_{L}\right)$ stable and in fact leaves every simple component of $\mathcal{C}^{+}\left(f_{L}\right)$ stable. We also use $\iota$ to denote the restriction of $\iota$ to $\mathfrak{C}^{+}\left(f_{L}\right)$ or to any of its components.

If $U$ is a semisimilitude of $f_{L}$ of ratio $\gamma$, then $U$ defines a semilinear automorphism $\alpha(U)$ of $\mathfrak{c}^{+}\left(f_{L}\right)$. If $U$ is $s$-linear $(s \in G)$ and $x_{1}, \ldots, x_{2 u}$ are $f_{L}$-orthogonal, then $\left(x_{1} \cdots x_{2 u}\right) \alpha(U)=\gamma^{-u}\left(x_{1} U\right) \cdots\left(x_{2 u} U\right)$. If $s=1$, so $U$ is linear, then $U$ is called proper or improper depending on whether $\alpha(U)$ leaves the center of $\mathfrak{C}^{+}\left(f_{L}\right)$ pointwise fixed or not. It is clear that every $\alpha(U)$ commutes with $\iota$.

Let $t(s)$ be the $s$-linear similitude of $f_{L}$ of ratio $\gamma(s)$ which was introduced in $\S 2.2$. $t(s) t(h)=t(s h) \delta(s, h)$ for $s, h \in G$ implies that $\alpha(t(s h))=\alpha(t(s)) \alpha(t(h))$. It follows from this that we may equip $\left(\mathbb{S}^{+}\left(f_{L}\right), \iota\right)$ with the structure of a $G$-algebra (with involution) by setting $X^{s}=X \alpha(t(s))$. The complete set of fixed points under $G$ is a $k$-algebra form (as algebra with involution) which we denote as $\mathfrak{C}^{+}(f)$ and which we call the even Clifford algebra of $f$. This is the algebra which Jacobson writes as $\mathfrak{C}^{+}(\mathfrak{A}, J)$ in [2].

If $U \in O^{+}\left(f_{L}\right)$ (the group of proper $f_{L}$-orthogonal transformations of $V$ ), then by examining the possible structures for $\mathcal{C}^{+}\left(f_{L}\right)$, we see that $\alpha(U)$ is an inner automorphism. We can realize $\alpha(U)$ as a conjugation in a manner consistent with the above action of $G$ since we have the following

LEMMA 2. If $U \in O^{+}\left(f_{L}\right)$, then we can choose $z(U) \in \mathbb{C}^{+}\left(f_{L}\right)$, where $X \alpha(U)=$ $z(U)^{-1} X z(U)$ for all $X \in \mathbb{C}^{+}\left(f_{L}\right)$ and which satisfies the condition, $z(U)^{s}=z\left(U^{s}\right)$.

Proof. The proof is similar to that of Lemma 3.2 of [3], but is somewhat more complicated. Let $\mathfrak{c}\left(f_{L}\right)$ be the center of $\mathfrak{c}^{+}\left(f_{L}\right)$, and let $\alpha^{\prime}(s)=\alpha(t(s)) \mid \mathfrak{c}\left(f_{L}\right)$. We will 
first show that $\left\{\alpha^{\prime}(s)\right\}$ is linearly independent with respect to right multiplications. If $c\left(f_{L}\right)$ is a field then this follows from Dedekind's independence theorem since our set consists of distinct automorphisms. Thus suppose that $\mathfrak{c}\left(f_{L}\right)$ is the diagonal algebra $L e_{1}+L e_{2}$. Since each $\alpha^{\prime}(s)$ is a ring automorphism it follows that $\alpha^{\prime}(s)$ either fixes each $e_{i}$, or exchanges them. Let $S$ be the subgroup of $G$ corresponding to the stabilizer of $e_{1}$ (hence also $e_{2}$ ). The $S$ is normal and of index at most 2 in $G$, and we let $G=S \cup h S$ be the (possibly trivial) coset decomposition.

Now suppose that $\left\{\alpha^{\prime}(s)\right\}$ is dependent with respect to right multiplications and let

$$
0=\sum_{s_{1} \in \mathscr{L}_{1}} \alpha^{\prime}\left(s_{1}\right) c_{s_{1}}+\sum_{s_{2} \in \mathscr{L}_{2}} \alpha^{\prime}\left(s_{2}\right) c_{s_{2}}, \quad \mathscr{L}_{1} \subset S, \mathscr{L}_{2} \subset h S, \mathscr{L}_{1} \cup \mathscr{L}_{2} \neq \varnothing
$$

be a dependence relation of minimal total length so that $c_{s_{i}}=c_{1}\left(s_{i}\right) e_{1}+c_{2}\left(s_{i}\right) e_{2} \neq 0$. Applying this relation to $\sigma e_{1}$, and then to $\sigma e_{2}, \sigma \in L$, we obtain

$$
\begin{aligned}
& 0=\sum_{s_{1}} \sigma^{s_{1}} c_{1}\left(s_{1}\right) e_{1}+\sum_{s_{2}} \sigma^{s_{2}} c_{2}\left(s_{2}\right) e_{2}, \\
& 0=\sum_{s_{1}} \sigma^{s_{1}} c_{2}\left(s_{1}\right) e_{2}+\sum_{s_{2}} \sigma^{s_{2}} c_{1}\left(s_{2}\right) e_{1} .
\end{aligned}
$$

Clearly these relations imply that each of $\mathscr{L}_{1}$ and $\mathscr{L}_{2}$ is a dependent set of automorphisms of $L$. Again by Dedekind's theorem this is possible if and only if $\mathscr{L}_{1} \cup \mathscr{L}_{2}=\varnothing$. Thus we obtain the stated independence of $\left\{\alpha^{\prime}(s)\right\}$.

For the moment fix $U \in O^{+}\left(f_{L}\right)$ and choose $Z_{s}$ in $C^{+}\left(f_{L}\right)$ with $X \alpha\left(U^{s}\right)=Z_{s}^{-1} X Z_{s}$. We show next that $\alpha\left(U^{s}\right)$ is conjugation by $Z_{e}^{s}$. Indeed if $x_{1}, \ldots, x_{2 u}$ are $f_{L}$-orthogonal elements of $V$, then

$$
\begin{aligned}
x_{1} \cdots x_{2 u} \alpha\left(U^{s}\right) & =x_{1} U^{s} \cdots x_{2 u} U^{s} \\
& =\left(x_{1} t(s)^{-1} U t(s)\right) \cdots\left(x_{2 u} t(s)^{-1} U t(s)\right) \\
& =\gamma(s)^{u}\left(x_{1} t(s)^{-1} U\right) \cdots\left(x_{2 u} t(s)^{-1} U\right)^{s} \\
& =\gamma(s)^{u}\left(Z_{e}^{-1} \cdot x_{1} t(s)^{-1} \cdots x_{2 u} t(s)^{-1} \cdot Z_{e}\right)^{s} \\
& =\left(Z_{e}^{-1}\right)^{s} \cdot x_{1} \cdots x_{2 u} \cdot Z_{e}^{s} .
\end{aligned}
$$

Thus $Z_{e}^{s}=w_{s, U} Z_{s}$ where $w_{s, U}$ is a unit in $\mathrm{c}\left(f_{L}\right)$. It is clear that $w_{s h, U}=w_{s, U}^{h} w_{h, U}$.

By the above independence, there is a $\kappa \in \mathfrak{c}\left(f_{L}\right)$ where $a=\sum \kappa^{s} w_{s, U^{s-1}}$ is invertible. If $\mathfrak{c}\left(f_{L}\right)$ is a field then this is clear. Thus suppose that we are dealing with the diagonal algebra $L e_{1}+L e_{2}$ as before. If the claim is false, then $\nu=\sum \alpha^{\prime}(s) w_{s, U^{s-1}}$ must be of rank 1 with either $e_{1}$ or $e_{2}$ as eigenvector. By symmetry, we only consider the first case. Write

Then

$$
\nu=\sum_{s \in S} \alpha^{\prime}(s) w_{s, U^{s-1}}+\sum_{s \in h S} \alpha^{\prime}(s) w_{s, U^{s-1}}
$$

$$
\left(\sigma e_{1}\right) \nu=\sum_{s \in S} \sigma^{s} w_{1}\left(s, U^{s^{-1}}\right) e_{1}+\sum_{s \in h S} \sigma^{s} w_{2}\left(s, U^{s^{-1}}\right) e_{2}
$$


$\left(w=w_{1} e_{1}+w_{2} e_{2}\right)$. Since $\left(\sigma e_{1}\right) \nu \subset L e_{1}$ and each $w_{s, U^{s-1}}$ is a unit we conclude that $h S=\varnothing$. But now the same reasoning applied to $\sigma e_{2}$ implies that $G$ is right dependent -a contradiction. This implies the existence of a $\kappa$ for which the corresponding $a$ is invertible. If we compute $a^{s}$ we obtain $\left(\sum_{h} \kappa^{h s} w_{h s, U} h^{-1}\right) w_{s, U}^{-1}$, which we write as $a_{s} w_{s, U}^{-1}$. Thus $a_{s}$ is invertible and if we set $z\left(U^{s}\right)=a_{s} Z_{s}$, then $z\left(U^{s}\right)^{h}=z\left(U^{s h}\right)$. We can thus establish the result for each of the (disjoint) orbits of $G$ in $O^{+}\left(f_{L}\right)$. This is enough. Q.E.D.

3.2. For the remainder of the paper we assume that we have made a choice of $z(U) \in \mathfrak{C}^{+}\left(f_{L}\right)$ which satisfy the requirements of the preceding lemma. For $U, V \in O^{+}\left(f_{L}\right), \alpha(U V)=\alpha(U) \alpha(V)$ so $z(U V)=\pi(U, V) z(U) z(V)$ where $\pi(U, V)$ is a unit in $\mathfrak{c}\left(f_{L}\right)$. Let $f_{1} \in(L, f)^{+}$. By Lemma 1 the cohomology class $c_{L}\left(f, f_{1}\right)$ is represented by a 1-cocycle $s \rightarrow U_{s}$ where $U_{s} \in O^{+}\left(f_{L}\right)$. A straightforward verification shows that

$$
(s, h) \rightarrow \pi\left(U_{h}, U_{s}^{h}\right)=z\left(U_{s h}\right)\left(z\left(U_{h}\right) z\left(U_{s}\right)^{h}\right)^{-1}
$$

is a 2-cocycle of $G$ in $\mathfrak{c}^{*}\left(f_{L}\right)$ where $\mathfrak{c}^{*}\left(f_{L}\right)$ is the group of units in $\mathfrak{c}\left(f_{L}\right)$. This correspondence induces a mapping on cohomology sets $\phi: \mathscr{H}_{+}^{1}(L, f) \rightarrow \mathscr{H}^{2}\left(G, \mathrm{c}^{*}\left(f_{L}\right)\right)$ and we set $a_{L}\left(f, f_{1}\right)=c_{L}\left(f, f_{1}\right) \phi$. In Hermitian forms. II, we show that when $f$ is a form of maximal Witt index in an even number of variables, then the above cohomology class represents an element of the Brauer group over $k$. In general it corresponds to a separable associative algebra over $k$ whose center is two dimensional. By applying ` to (9) and remembering that ı commutes with every $\alpha(U)$ we see that $a_{L}\left(f, f_{1}\right)$ has order at most two.

THEOREM 4. $a_{L}\left(f, f_{1}\right)=1 \Rightarrow \mathfrak{C}^{+}(f) \cong \mathfrak{C}^{+}\left(f_{1}\right)$.

Proof. It is enough to show that there is a $G$-isomorphism between $\mathfrak{C}^{+}\left(f_{1 L}\right)$ and $\mathfrak{C}^{+}\left(f_{L}\right)$. Let $s \rightarrow U_{s}$ be a 1-cocycle whose cohomology class is $c_{L}\left(f, f_{1}\right)$. Then $a_{L}\left(f, f_{1}\right)=1$ implies that there are $\zeta_{s} \in \mathrm{c}^{*}\left(f_{L}\right)$ with $z\left(U_{s h}\right)\left(z\left(U_{h}\right) z\left(U_{s}^{h}\right)\right)^{-1}=\zeta_{h} \zeta_{s}^{h} \zeta_{s h}{ }^{-1}$. We set $b_{s}=z\left(U_{s}\right) \zeta_{s}$ and note that $s \rightarrow b_{s}$ is a 1-cocycle. By a result of Springer, [3, appendix] there is an invertible element $b$ of $\mathfrak{c}^{+}\left(f_{L}\right)$ with $b_{s}=b^{-1} b^{s}$. Thus $X \alpha\left(U_{s}\right)=b_{s}^{-1} X b_{s}=\left(b^{-1}\right)^{s} b X b^{-1} b^{s}$.

Let $T$ be an equivalence between $f_{L}$ and $f_{1 L}$ with $T^{s}=T U_{s}$. We have an isomorphism $\lambda: \mathfrak{S}\left(f_{1 L}\right) \rightarrow \mathfrak{E}\left(f_{L}\right)$ such that for $x \in V, x \lambda=x T . \lambda$ necessarily sends $\mathfrak{C}^{+}\left(f_{1 L}\right)$ onto $\mathfrak{C}^{+}\left(f_{L}\right)$ and we define $\Theta: \mathfrak{C}^{+}\left(f_{1 L}\right) \cong \mathfrak{C}^{+}\left(f_{L}\right)$ by $X^{\boldsymbol{\theta}}=b X^{\lambda} b^{-1}$.

If $x_{1}, \ldots, x_{2 r}$ are $f_{L}$-orthogonal, then

$$
\begin{aligned}
\left(x_{1} \cdots x_{2 r}\right)^{\theta s} & =\gamma_{(s)}^{-r} b^{s}\left(x_{1} T t(s)\right) \cdots\left(x_{2 r} T t(s)\right)\left(b^{-1}\right)^{s} \\
& =\gamma_{(s)}^{-r} b^{s}\left(x_{1} t(s) T U_{s}\right) \cdots\left(x_{2 r} t(s) T U_{s}\right)\left(b^{-1}\right)^{s} \\
& =\gamma_{(s)}^{-r} b(x t(s) T) \cdots\left(x_{2 r} t(s) T\right) b^{-1} \\
& =\left(x_{1} \cdots x_{2 r}\right)^{s \theta} .
\end{aligned}
$$

Thus $\Theta$ is a $G$-isomorphism. Q.E.D. 
As our final result we establish a relationship between several $a_{L}\left(f, f_{1}\right)$ 's.

TheOREM 6. Suppose that $a_{L}\left(f, f_{1}\right)$ and $a_{L}\left(f, f_{2}\right)$ are defined. Then $a_{L}\left(f_{1}, f_{2}\right)$ is defined and we have an isomorphism $\sigma: \mathscr{H}^{2}\left(G, \mathrm{c}^{*}\left(f_{1 L}\right)\right) \cong \mathscr{H}^{2}\left(G, \mathrm{c}^{*}\left(f_{L}\right)\right)$ such that $a_{L}\left(f_{1}, f_{2}\right)^{\sigma}=a_{L}\left(f, f_{1}\right) a_{L}\left(f, f_{2}\right)$.

Proof. Let $f_{L}\left(x T_{i}, y T_{i}\right)=f_{i L}(x, y), i=1,2$ with $T_{1}^{s}=T_{1} U_{s}, T_{2}^{s}=T_{2} V_{s}$.

$$
f_{1 L}\left(x T_{2} T_{1}^{-1}, y T_{2} T_{1}^{-1}\right)=f_{2 L}(x, y)
$$

so we can compute $c_{L}\left(f_{1}, f_{2}\right)$ by means of $T_{2} T_{1}^{-1} . \quad\left(T_{2} T_{1}^{-1}\right)^{s}=T_{2} T_{1}^{-1} W_{s}$, $W_{s}=T_{1} V_{s} U_{s}^{-1} T_{1}^{-1}$. Since determinant is a class function and $U_{s}$ and $V_{s}$ are proper, it follows that $W_{s}$ is proper so $a_{L}\left(f_{1}, f_{2}\right)$ is defined. $T_{1}$ defines an isomorphism $\Theta: \mathfrak{S}^{+}\left(f_{1 L}\right) \cong \mathfrak{C}^{+}\left(f_{L}\right)$ which is induced by the map $x \in V \subseteq \mathbb{S}\left(f_{1 L}\right) \rightarrow x T_{1} \in \mathbb{C}\left(f_{L}\right)$. If $x_{1}, \ldots, x_{2 r}$ are $f_{1 L}$-orthogonal, then $\left(x_{1} \cdots x_{2 r}\right)^{\tilde{\alpha}\left(W_{s}\right) \theta}=\left(x_{1} W_{s} T_{1}\right) \cdots\left(x_{2 r} W_{s} T_{1}\right)$. But $\left(x_{1} \cdots x_{2 r}\right)^{\bar{\alpha}\left(W_{s}\right) \Theta}=\left(\bar{z}\left(W_{s}\right)^{-1} x_{1} \cdots x_{2 r} \bar{z}\left(W_{s}\right)\right)^{\Theta}=\left(\bar{z}\left(W_{s}\right)^{\Theta}\right)^{-1}\left(x_{1} T_{1}\right) \cdots\left(x_{2 r} T_{1}\right) \bar{z}\left(W_{s}\right)^{\Theta}$. For $y_{1}, \ldots, y_{2 r} f_{L}$-orthogonal, this gives

$$
\begin{aligned}
\left(\bar{z}\left(W_{s}\right)^{\Theta}\right)^{-1} y_{1} \cdots y_{2 r} \bar{z}\left(W_{s}\right)^{\Theta} & =\left(y_{1} T_{1}^{-1} W_{s} T_{1}\right) \cdots\left(y_{2 r} T_{1}^{-1} W_{s} T_{1}\right) \\
& =\left(y_{1} V_{s} U_{s}^{-1}\right) \cdots\left(y_{2 r} V_{s} U_{s}^{-1}\right) \\
& =z\left(U_{s}\right) z\left(V_{s}\right)^{-1} y_{1} \cdots y_{2 r} z\left(V_{r}\right) z\left(U_{s}\right)^{-1}
\end{aligned}
$$

This relation shows that there exists $\varepsilon_{s} \in \mathrm{c}^{*}\left(f_{L}\right)$ with

$$
\bar{z}\left(W_{s}\right)^{\Theta}=\varepsilon_{s} z\left(V_{s}\right) z\left(U_{s}\right)^{-1} .
$$

Also

$$
\begin{aligned}
\left(x_{1} \cdots x_{2 r}\right)^{\Theta s} & =\gamma_{(s)}^{-r}\left(x_{1} T_{1} t(s)\right) \cdots\left(x_{2 r} T_{1} t(s)\right) \\
& =\gamma_{(s)}^{-r}\left(x_{1} t(s) T_{1} U_{s}\right) \cdots\left(x_{2 r} t(s) T_{1} U_{s}\right) \\
& =z\left(U_{s}\right)^{-1}\left(x_{1} \cdots x_{2 r}\right)^{s \theta} z\left(U_{s}\right) .
\end{aligned}
$$

Our last equation shows that $X^{\theta s}=z\left(U_{s}\right)^{-1} X^{s \theta} z\left(U_{s}\right)$ for all $X$ in $\widetilde{c}^{+}\left(f_{L}\right)$. This implies that the restriction of $\Theta$ to $c^{*}\left(f_{1 L}\right)$ is a $G$-isomorphism onto $c^{*}\left(f_{L}\right)$ and thus induces an isomorphism $\sigma: \mathscr{H}^{2}\left(G \mathrm{c}^{*}\left(f_{1 L}\right)\right) \cong \mathscr{H}^{2}\left(G, \mathrm{c}^{*}\left(f_{L}\right)\right) . a_{L}\left(f_{1}, f_{2}\right)^{\sigma}$ is the cohomology class of $(s, h) \rightarrow\left\{\bar{z}\left(W_{s h}\right)\left(\bar{z}\left(W_{h}\right) \bar{z}\left(W_{s}^{h}\right)\right)^{-1}\right\}^{\Theta}$ while $a_{L}\left(f, f_{1}\right)$ and $a_{L}\left(f, f_{2}\right)$ are the cohomology classes of $(s, h) \rightarrow z\left(U_{s h}\right)\left(z\left(U_{h}\right) z\left(U_{s}^{h}\right)\right)^{-1}$ and $(s, h) \rightarrow$ $z\left(V_{s h}\right)\left(z\left(V_{h}\right) z\left(V_{s}^{h}\right)\right)^{-1}$ respectively.

From (10) we immediately obtain

$\left.\left(\bar{z}\left(W_{s h}\right)\left(\bar{z}\left(W_{h}\right) \bar{z}\left(W_{s}^{h}\right)\right)^{-1}\right)^{\Theta}=\varepsilon_{h} \varepsilon_{s}^{h}{\varepsilon_{s h}^{-1}}^{-1}\left(U_{s h}\right)\left(z\left(U_{h}\right) z\left(U_{s}^{h}\right)^{-1}\right)\right)^{-1}\left(z\left(U_{s h}\right)\left(z\left(V_{h}\right) z\left(V_{s}^{h}\right)\right)^{-1}\right)$.

Thus $a_{L}\left(f_{1}, f_{2}\right)^{\sigma}=a_{L}\left(f_{1}, f\right)^{-1} a_{L}\left(f, f_{2}\right)=a_{L}\left(f, f_{1}\right) a_{L}\left(f, f_{2}\right)$ since our cohomology classes are of order at most 2. Q.E.D.

Corollary 1. $a_{L}\left(f, f_{1}\right)$ if defined depends only on the cohomology class of $f$ and $f_{1}$.

Corollary 2. If $a_{L}\left(f, f_{1}\right)=a_{L}\left(f, f_{2}\right)$, then $\mathfrak{E}^{+}\left(f_{1}\right) \cong \mathfrak{C}^{+}\left(f_{2}\right)$. 


\section{BIBLIOGRAPHY}

1. A. A. Albert, Structure of algebras, Amer. Math. Soc. Colloq. Publ., Vol. 24, Amer. Math. Soc., Providence, R. I., 1961.

2. N. Jacobson, Clifford algebras for algebras with involution of type D, J. Algebra 1 (1964), 288-300.

3. T. A. Springer, On the equivalence of quadratic forms, Proc. Kon. Nederl. Akad. Wetensch. Ser. A 69 (1959), 241-253.

Massachusetts Institute of Technology,

Cambridge, Massachusetts

RIJKSUNIVERSITEIT TE UTRECHT,

UtreCht, NetherLaNDS 